Physlological Imaging

28.4-1

\title{
MODELING OF HIGH RESOLUTION DIGITAL RETINAL IMAGING
}

\author{
Artur V. Cideciyan ${ }^{1,2}$, Joachim H. Nagel', Samuel G. Jacobson' \\ 'Department of Ophthalmology, Bascom Paimer Eye Institute, School of Medicine \\ ${ }^{2}$ Department of Biomedical Engineering, College of Engineering \\ University of Miami, Miami, Florida, USA
}

\begin{abstract}
High resolution digital images of the retina can be obtained by photography with a Zeiss fundus camera followed by digitization of the photographic slide with a high resolution scanner. A complete model of this imaging system is developed based on its four components; the eye, the camera, the film and the scanner. The actual and modeled step responses and system noise are compared to validate the model. A simulated retinal reflection is used to demonstrate the extent of information degradation caused by such an imaging system. Preliminary results of linear restoration using a simplified version of the complete model are given. Development of nonlinear restoration incorporating the complete model is in progress.
\end{abstract}

\section{INTRODUCTION}

Photography of the eyes of ophthalmic patients is a commonly used and valuable clinical technique to document eye disease. The recent explosion of computing resources has made it feasible to use digital image processing techniques to increase the amount of usable information from ocular photographs. It is now also possible to quantify some of the abnormalities detected in these photographs. Applications of digital image processing have mainly concentrated on the images of the fundus (the inside, back portion of the eye). Some examples include the enhancement of images for increased visibility of features, deblurring of images taken through cataractous lenses, segmentation of blood vessels, hemorrhages, exudates and drusen, photogrammetry for the quantitative analysis of optic nerve head cup volume, and on-line measurement of retinal blood flow from fluorescein angiograms [1]. Although many investigators have explored the exciting possibilities digital image processing can offer, little attention has been paid to the characterization of the imaging system that produces the digital data. In this paper, we develop a thorough model of a high resolution digital retinal imaging system that can be implemented in any laboratory with relatively low cost.

We preferred the most common approach of image acquisition; scanning existing $35 \mathrm{~mm}$ color transparencies with a high resolution scanner. This 'composite' setup is not only cost-effective but also allows the use of the immense database of photographs taken over the years for patient follow-up studies. The alternative, direct digital fundus imaging, is possible either by mounting a CCD to the fundus camera in place of a film, or by using a scanning-laserophthalmoscope (SLO). Both of these methodologies currently suffer from low resolution and/or high cost as compared to the method used in this paper, but probably achieve higher SNR and better quantitation. In this paper, a complete model of the digital retinal imaging system is developed by considering four separate components involved; the eye, the camera, the film, and the scanner. The validity of the model is verified by comparing measured and modeled total system noise and system step response. With the aim of giving the reader a feeling for the extent of the degradation, a plausible pattem of retinal reflectance is simulated and the model is used to calculate the expected digital image. Preliminary results of linear restoration of both the simulated and the actual retinal images are shown.

\section{MODELING}

The four components of the digital retinal imaging system are shown schematically in Figure 1. Each one of the four components is modeled separately (not necessarily independently) before being combined into a single complete model.

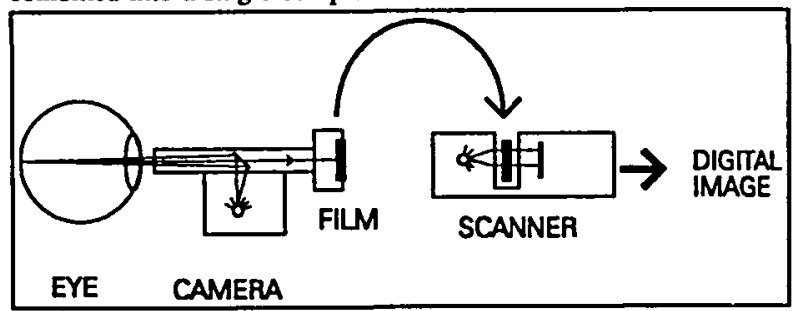

Figure 1: Schematic diagram of high resolution digital fundus imaging system. Illuminating light originates inside the camera, travels to the fundus, and it is partially reflected back. The reflected light is imaged onto a photographic film, which is digitized with a high resolution scanner.

\section{The Human Eye}

The light reflected from the fundus has to travel through many semi-transparent layers of the human eye before exiting through the pupil. Scattering through the various layers causes blurring of the spatial information content. For a small region of interest, the blurring can be modeled as a linear, spatially invariant and isotropic system. Representative point spread functions (PSF) have been published for typical human eyes [2]. The PSFs are usually specified as a function of the pupil diameter. Due to the collection optics of the fundus camera we assume an effective pupil diameter of $1.5 \mathrm{~mm}$ and we use a corresponding modulation transfer function (MTF) [2]. MTF is the magnitude of the Fourier transform of the PSF. Considering the PSF is rotationally symmetric, definition of a linear system by the MTF is equivalent to the defintion by the PSF.

\section{The Fundus Camera}

A Zeiss FF4 fundus camera was used in-this work. Fundus cameras are special telescopes of low magnification $(2.5 \mathrm{X})$ that use an aspherical lens to image a hemispherical surface (the fundus) onto a planar detector (photographic film). Fundus cameras have been considered to be the main resolution limiting component in a fundus photography system [3]. The only information available about the Zeiss fundus camera is that it has resolutions of $8 \mu \mathrm{m}$ and $15 \mu \mathrm{m}$, as measured on the fundus, at the center of its optical axis and at the periphery, respectively. The blurring introduced by the fundus camera can be modeled as a linear system with a spatially variant PSF. Furthermore, for a small region of interest PSF can be assumed to be spatially invariant and isotropic. As the shape of the fundus camera PSF is not known a functional form is assumed that is both reasonable and mathematically simple. The corresponding MTF shape is modified so that the specified camera resolution corresponds to 10 percent modulation. 


\section{The Film}

Fujichrome 100 RD color slide film was used for all fundus photographs. This is a medium resolution, medium noise film commonly used for clinical purposes. Film is a detector that converts light energy to optical density. The exposure to density mapping process is usually modeled as linear blur of exposing light followed by a nonlinear point process with the addition of signal dependent noise [4]. The effective exposure MTF and the nonlinear exposure to density mapping are film specific and are given by the film manufacturer. The film-grain noise is specified by the film manufacturer at standard measuring conditions which is extrapolated to the conditions used in this work.

\section{The Scanner}

The color slides were scanned with a Nikon LS3500 Slide Scanner. This scanner uses a stationary linear array of 4096 CCDs and a stationary light source. The slide is moved mechanically between the light and the detector array. The effective scanning aperture (pixel size) is a square of $6 \mu \mathrm{m}$ side. The scanner maps the information of optical density embedded in the film to a transmittance value. The scanner can be modeled as a nonlinear point process mapping density to transmittance, followed by a linear isotropic space-invariant blurring in the transmittance space and additive signal dependent noise [5]. All of the scanner characteristics were determined experimentally using uniform density filters for the nonlinear mapping and noise parameters and a very sharp ( $<3 \mu \mathrm{m}$ ) density 'step' for bluming determination.

\section{The Complete Model}

Figure 2 shows radial sections of the isotropic MTFs for all four systems in terms of spatial frequency measured on the film plane. It is important to keep in mind that the MTFs for the eye, the fundus camera and the film act in the exposure domain and can be combined into a single MTF by simple multiplication in the frequency domain.

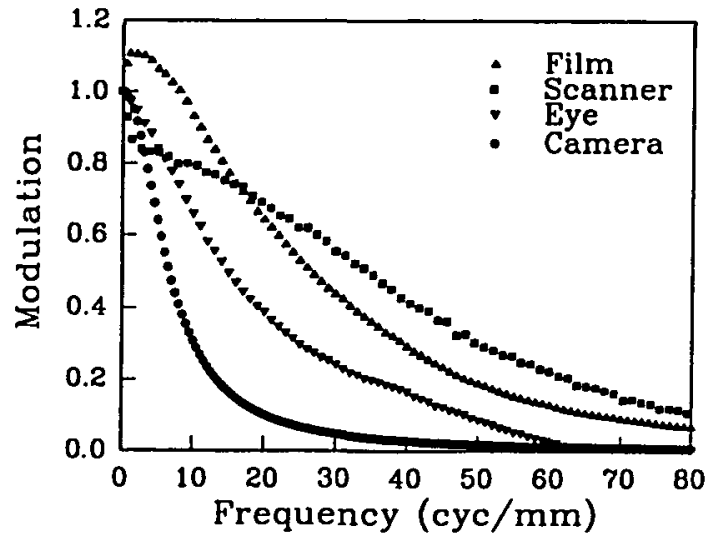

Figure 2: Radial sections from the isotropic MTFs of the four systems.

The resulting imaging model equations are

$$
\begin{gathered}
t=s_{1}[d] * h_{\infty}+s_{2}[d] n_{1} \\
d=s_{1}\left[e * h_{s 00}\right]+s_{4}\left[e * h_{d j_{0}}\right] n_{2}
\end{gathered}
$$

where

t is the digital transmission image.

$d$ is the film density,

c is the exposure function due to reflection from the retina,

$n_{1}, n_{2}$ are independent Gaussian noise sources with zero mean and unit variance,

$h_{\text {sen }} \quad$ is the scanner PSF,

$h_{r *}$ is the combination of PSFs due to eye, camera and film.
The four nonlinear functions of (1) and (2) are $s_{1}[v]=10 \frac{2716-0}{104}$, the scanner point nonlinearity $s_{2}[v]=0.231+0.044 \sqrt{10 \frac{2714-v}{1044}}$, signal dependence of scanner noise $s_{3}[v]$ is the nonlinear mapping from exposure to density that can be extracted from the D-logE curve published by the film manufacturer $s_{4}[v]=0.078 \sqrt{s_{3}[v]}$, signal dependence of the film-grain noise

\section{Model Validation}

The models' validity was verified in two independent steps. First, nine uniformly exposed slides at different densities were digitized. The mean value and the standard deviation of quantized intensities were measured in eight $256 \times 256$ subregions of each uniformly exposed slide. These 72 pairs of values showing the signaldependence of the total system noise are plotted in Figure 3. A comparison is made with mean and standard deviation values of simulated uniform density images where noise was added using pseudo-random number generators according to the imaging model. Figure 3 clearly shows the close correspondence of the actual system noise and the expected noise due to our model.

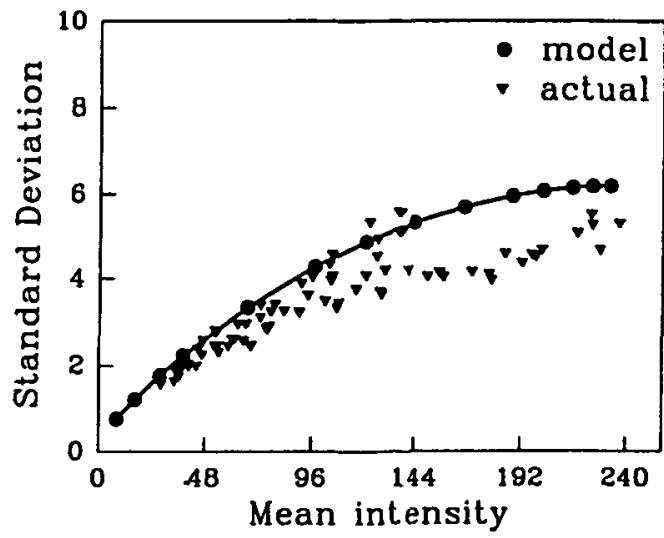

Figure 3: Validation of the noise madel.

The second step of validation was the verification of the blurring caused by three systems (camera, film and scanner) by photographing and digitizing a vertical sharp reflection 'step'. Consecutive digitized step profiles were averaged to obtain a smooth estimate of the system step response (cross-correlation was used to register the profiles before averaging). The line-spread-function (LSF) was calculated by differentiating the smooth step response. LSF of a simulated step, degraded according to our model (not including the bluring due to the eye) was comparable to the actually measured LSF.

\section{Simulation}

The model of digital fundus imaging as specified by equations (1) and (2), gives very little intuitive feeling about the extent of the degradation of information. Therefore, we simulated a realistic reflection function composed of differently sized patches of $20 \%$ reflectance on a background of $10 \%$ reflectance. The illumination was simulated to be a function that slowly varies about a mean level. The degraded output of the imaging system was calculated using an exposure function which is the multiplication of illumination and reflection functions and the exposure time. Figure 4 shows the simulated exposure function and the degraded transmittance image. The edges of large structures are blurred, small structures almost completely vanish and there is signal dependent noise in the image. 


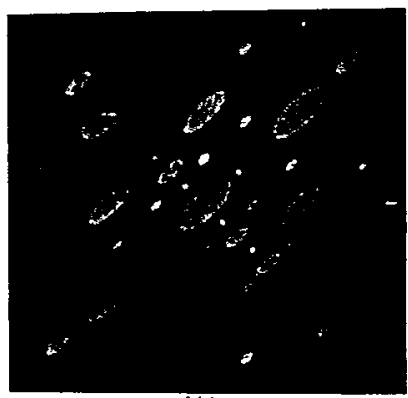

(A)

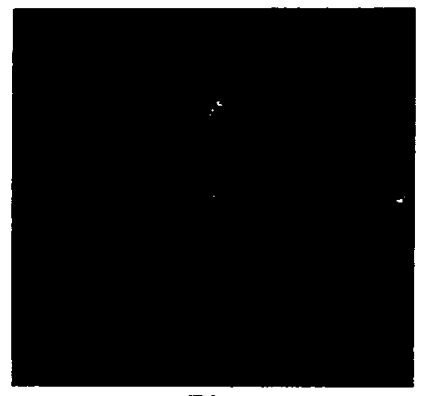

(B)

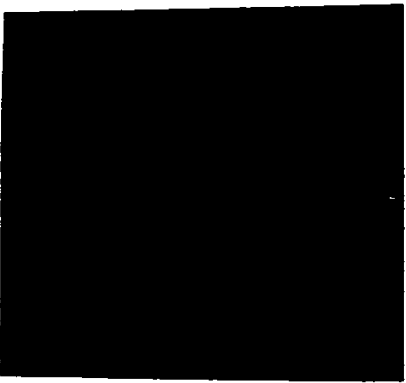

(C)

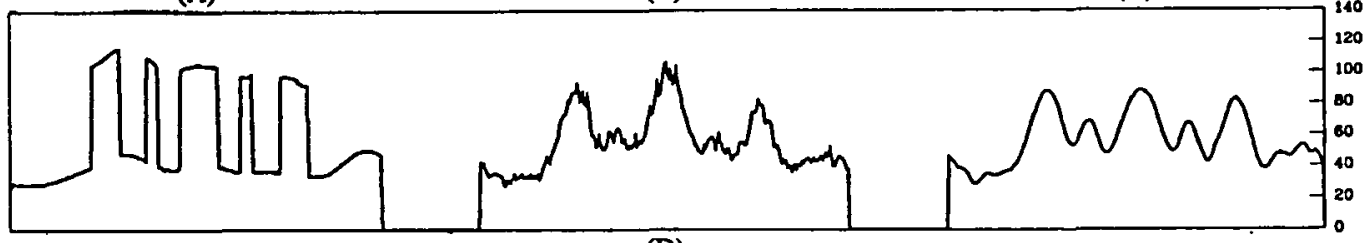

(D)

Figure 4: (A)Simulation of a digitized plausible retinal reflection function. (B)Simulated function degraded according to the complete imaging model (C)Restoration of the degraded simulation with a linear restoration (D)Profile of pixel intensities at level of the small arrow on the images.

\section{RESTORATION}

The main goal of our research is the analysis of retinal images showing tiny reflections unique to the carrier state of a blinding disease, X-linked retinitis pigmentosa [6]. The reduction of noise and simultaneous sharpening of small blurred structures is paramount to our very high resolution application. Unfortunately, the two nonlinearities and the two signal dependent noise sources in our model make restoration a nontrivial problem. Therefore, as a first step, we simplified the complete model to linear bluning and signal independent noise. This simple model was used with a constrained least squares restoration method [7]. The restoration of the simulated retinal reflection can be seen in Figure $4 \mathrm{C}$. The remaining difference between the restored image (Figure 4C) and the original image (Figure $4 A$ ) indicates that there is no perfect restoration. On the other hand, comparing the restored image to the degraded image we see that even our simplistic restoration achieves good noise suppression and simultaneous enhancement of small structures previously blurred out. An example of an actual retinal image and its restoration with the constrained least squares method are shown in Figure 5.

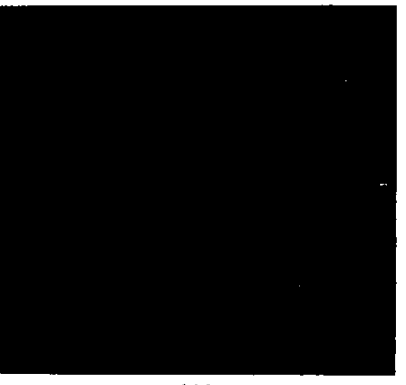

(A)

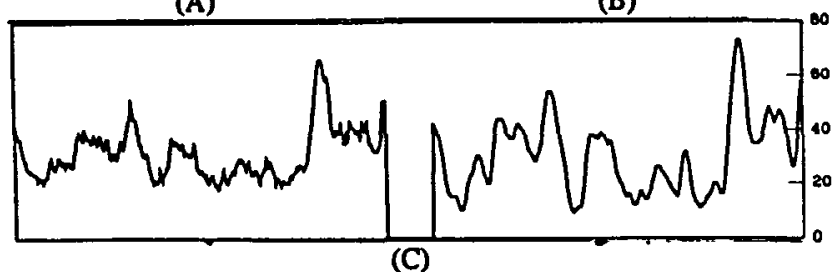

Figure 5: (A)Actual digitized $400 \times 400$ subregion of a high resolution retinal image (B)Restored retinal image, (C)Profile of pixel intensities at level of the small arrow on the images.

\section{CONCLUSION}

In this paper, we developed and validated a complete model of a high resolution digital retinal imaging system. We showed how the model can be used in attempts of restoration, i.e. the estimation of the underiying scene that gave rise to the image. In addition, our model may be valuable to many other image processing approaches in ophthalmology as it shows the degradation of information in digital images of the retina. Any simplifying assumptions, forced by mathematical tractability, can be based on our complete model instead of being ad hoc.

Our efforts are currently concentrated on the incorporation of model nonlinearities and signal-dependent noise in the restoration.

\section{ACKNOWLEDGMENT}

We gratefully acknowledge helpful contributions of Dr R.W. Knighton and Dr C. M. Kemp. This research was supported in part by the National Retinitis Pigmentosa Foundation, Inc. (Baltimore, MD).

\section{REFERENCES}

[1] J. Gilchrist, "Computer Processing of Ocular Photographs - A Review", Ophthal. Physiol. Opt., 7(4):379-386, 1987.

[2] R.W. Gubisch, "Optical Performance of the Human Eye", JOSA, 57(3):407-415, 1967.

[3] L. Frisen, "Resolution at Low Contrast with a Fundus Camera. Comparison of Various Pholographic Films", Invest. Ophthalmol., 12(11):865-869, 1973.

[4] H-C. Lee, "Review of Image-blur Models in a Photographic System using the Principles of Optics", Opt. Eng., 29(5):405-421, 1990.

[5] J.C. Dainty and R. Shaw, "Image Science", Academic Press, San Diego, 1988.

[6] A.V. Cideciyan, S.G. Jacobson, J.H. Nagel, "Multi-scale Segmentation of Retinal Images", 13th Int. Conf. of IEEE/EMBS, 1991 (submitted)

[7] B.R. Hunt, "The Application of Constrained Least Squares Estimation to Image Restoration by Digital Computer", IEEE Trans. Comp., C-22(9):805-812, 1973.

Mailing Address: Artur Cideciyan, McKnight Vision Research Center 1638 NW 10th Avenue, Miami, FL 33136, USA tel: (305)-326-6089, fax: (305)-326-6306 